\title{
Aproximación al estudio de la gerencia en las organizaciones del Tercer Sector,en el municipio San Francisco del estado Zulia*
}

\author{
Rodríguez Colmenares, I sabel $* *$ \\ Rodríguez López, J udith*** \\ Scarbay, Lisbeth**** \\ ** MgSc en Ciencias Políticas. Investigadora del CESA. E-mail: rcs luz@yahoo.com \\ *** MgSc en Desarrollo Social. Investigadora del CESA. E-mail: \\ judithrodriguez_ve@yahoo.com \\ **** Soc. Auxiliar de Investigación del CESA, cursante de la Maestría en Desarrollo \\ Social. E-mail: lisbethscarbay@hotmail.com
}

\section{Resumen}

En Venezuela, desde mediados de la década de los ochenta, surgen un gran número de organizaciones del Tercer Sector que desarrollan proyectos sociales en el ámbito local y asumen funciones de intermediación entre las demandas emergentes de la sociedad y las ofertas que efectúa el Estado; aspecto éste que se incrementa en los noventa. El objetivo del trabajo, es presentar una aproximación al estudio de la gerencia en las organizaciones del Tercer Sector proveedoras de servicios sociales ubicadas en el municipio San Francisco del estado Zulia en Venezuela. La investigación se adelantó durante el 2001, teniendo como base la revisión de fuentes documentales, bibliográficas y hemerográficas, así como la aplicación de entrevistas semiestructuradas a miembros de tres organizaciones seleccionadas atendiendo a: su ubicación geográfica, su trayectoria histórica y la condición de ser proveedoras de servicios en las áreas de salud, capacitación laboral y educación. Se encontró que estas organizaciones presentan una tendencia democratizadora, evidenciando algunas formas de participación, se realizan asambleas y reuniones para la toma de decisiones y acciones, algunas organizaciones, tienden hacia la descentralización, y se registra una modesta iniciativa en la formación de redes de cooperación para la consecución de bienestar en las respectivas localidades.

Palabras clave: Organizaciones del Tercer Sector, gerencia, servicios sociales, gestión social, estado Zulia.

\section{Approximations to a Study of Third Sector Organizations in San Francisco}




\title{
Municipality, Zulia State
}

\begin{abstract}
In Venezuela, since the mid-nineteen eighties, a large number of third sector organizations have emerged that develop social projects on a local level and which assume intermediary functions between emerging societal demanders and governmental offers: this aspect increased in the 1990s. The objective of this study is to present an approach towards studying the management of third sector organizations that provide social services in the San Francisco Municipality in Zulia State, Venezuela. The research was undertaken during 2001, based on a bibliographical review of documents, bibliography, and other publications, as well as on the application of semi-structured interviews of members of three organizations selected according to their geographic location, historic trajectory, and condition as providers of services in the areas health, labor and education. These organizations were found to present a tendency towards democratic functioning, evidenced by the forms of participation, the realization of assemblies and meeting in order to make decisions and undertake actions, and in some cases, the tendency towards decentralization. They registered a modest initiative towards the formation of cooperative networks in order to offer well-being to their respective localities.
\end{abstract}

Key words: Third sector organizations, management, social services, social management, Zulia State.

Recibido: 02-06-04. Aceptado: 02-12-10

\section{I ntroducción}

La complejidad del tercer sector tiene que ver con varios aspectos, entre los que destacan: la presencia de diversos tipos de organizaciones; su afiliación ideológica y doctrinaria, que puede recorrer desde posiciones de extrema derecha hasta de izquierda; los objetivos que persiguen y el rol que desempeñan en la sociedad, entre otros. En tal sentido, autores como Rizzardini (1997: 263), indican que en el llamado tercer sector hay de todo: lo sacro y lo profano; lo más virtuoso y lo más impresentable, porque así como encontramos las organizaciones de solidaridad nacionales e internacionales, grupos ecológicos, conservacionistas, fundaciones de apoyo a la infancia y otros, también se registra la presencia de sectas y organizaciones de índoles inconfesables, como el Ku Klux Klan 1 y otras sectas de tipo religiosas, que recaudan fondos con la finalidad de mantener 
sus posturas radicales xenofóbicas o las de índole religiosa que se presentan para hacer caridad en los pueblos de Latinoamérica utilizando canales privados de televisión por cable para recaudar fondos millonarios para la defensa de sus causas. Por otra parte, se encuentran las fundaciones que surgen de las grandes empresas trasnacionales y Holding, y otras de capital financiero que efectúan subastas fastuosas; se encuentran también las asociaciones civiles sin fines de lucro, organizaciones no gubernamentales, cooperativas y muchas otras tipos de organizaciones que se ocupan de actividades de carácter semiprivadas o las que atienden a grupos vulnerables de la población con rasgos asistencialistas y filantrópicos.

Reconociendo entonces la diversidad, nos ocupamos en esta investigación, de poner en foco algunos aspectos que caracterizan a las organizaciones que conforman el tercer sector ubicadas en el municipio San Francisco del estado Zulia, con la finalidad de tener una idea más próxima al funcionamiento de la gerencia con que se desempeñan. Esto representa afrontar un reto por cuanto se trata de penetrar un mundo tan complejo y variado, donde intervienen diferentes actores con modalidades de trabajo muy particulares y, porque además, los estudios que se han adelantado son descriptivos de la actuación de dichas organizaciones. Por lo cual se han centrado en los aspectos morfológicos, descuidando el análisis sobre aspectos como: los impactos culturales; su contribución al afianzamiento o no de los procesos democratizadores en América Latina, entre otros.

Aquí, sólo nos aproximamos a la presentación de pocos casos, por tanto las conclusiones son preliminares y sólo dan cuenta del registro que se ha efectuado hasta el momento. Se realizaron entrevistas semiestructuradas a miembros de las organizaciones, se les aplicó un cuestionario, se consultaron informes anuales, boletines, folletos y otras fuentes publicadas por estas organizaciones, donde aparece descrito su perfil y filosofía. Estas entrevistas se efectuaron en el período comprendido entre marzo del 2001 a marzo del 2002, registrándose originalmente alrededor de ciento setenta organizaciones de todo tipo, de la cuales para efectos de la ejecución del proyecto se seleccionaron cien que cumplían con las condiciones de estar activas, ser proveedoras de servicios sociales; estar operando efectivamente en el lapso bajo estudio; y estar ubicada en el municipio San Francisco del estado Zulia.

A los efectos de este trabajo optamos por presentar tres casos que fueron seleccionados en atención a la representatividad de las acciones que estas organizaciones reportan para los sectores más sensibles del ámbito social como son: educación, salud y capacitación laboral. De esta forma se ofrece una panorámica que recoge la diversidad de estas 
organizaciones.

Se presentan los casos correspondientes a las siguientes organizaciones que hacen vida en el municipio San Francisco del estado Zulia / Venezuela, desde a ños atrás:

- Equipo de Formación, I nformación y Publicaciones (EFI P), fundado en 1972; esta organización nace en Valencia Estado Carabobo, promovida inicialmente por el Movimiento de J óvenes Católicos.

- Asociación Civil Nuevo Amanecer, fundada en Maracaibo en 1985, esta organización se deriva del Centro al Servicio de la Acción Popular (CESAP) la cual a su vez nace en 1975 y su sede principal se encuentra ubicada en Caracas.

- Asociación Damas Salecianas, creada en Caracas en 1968 por el Padre Miguel González y tiene presencia a nivel nacional.

Cabe acotar, que si bien es cierto, estas organizaciones no nacieron en el municipio San Francisco, ellas fueron seleccionadas en atención a las siguientes razones; han desarrollado una actividad de trabajo manteniendo una relativa autonomía que les ha permitido ampliar su radio de acción; han consolidado y mantienen una línea de trabajo definida en el tiempo y han logrado resultados positivos en áreas como educación, salud y capacitación, cuyos impactos, si bien no han sido medidos hasta hoy, sus actividades son reconocidas en el ámbito regional.

Dentro de las distintas organizaciones se seleccionó una representativa del sector religioso, con la finalidad de resaltar el creciente rol que la Iglesia ha vendido teniendo vinculado a la provisión de servicios sociales en las áreas de salud y educación, lo que además evidencia la intervención de organizaciones eclesiales como la Arquidiócesis de Maracaibo en la gestión de servicios sociales tal como lo evidencian otros trabajos (Rodríguez y Pérez, 2000; García y Sarcos, 2000).

\section{Relación Estado-Sociedad Civil en Venezuela en la década de los ochenta}

En Venezuela a principio de la década de los '80, se presentan los primeros síntomas de la crisis económica, política y social, el Estado, pierde su capacidad para satisfacer el gran cúmulo de demandas sociales, lo cual afecta su legitimidad, en otros términos la oferta de servicios para la mayor parte de los ciudadanos, disminuyó en gran medida, presentándose la denominada crisis de gobernabilidad, dibujándose así, una alteración de la lógica de compromiso entre igualdad y desigualdad; la exclusión y la equidad; el acceso y la oportunidad a los beneficiarios que las distintas políticas sociales permitían, lo que 
condujo a la aceleración de dicha crisis. Es precisamente esta forma de compromiso entre igualdad y desigualdad, entre lógica del mercado y los derechos sociales de ciudadanía, entre leyes de la economía y solidaridad social, gestionada con la mediación del Estado; factores todos que según la opinión de algunos estudiosos, condujeron a la crisis que se vivió en dicha década (Salinas, 1997:111).

Esta crisis económica y social tiene como expresión en el ámbito político, entre otros aspectos, el agotamiento del sistema de pactos entre los principales actores políticos y la incapacidad del Estado de cumplir con la cantidad de demandas sociales; lo que lleva a su vez, a la redefinición del papel asumido por el mismo, así como el de la sociedad civil, ambos van a apelar a mecanismos de autorrecuperación; el primero, intenta reorientar la provisión de los servicios sociales para hacerlos más eficientes, eficaces y equitativos, para lo cual busca incorporar nuevos actores en la gestión de los programas sociales, mientras que la segunda, genera procesos de organización de los grupos sociales que intentan asumir funciones que anteriormente estaban en manos del Estado, como es la gestión del bienestar.

Esta redefinición de las fronteras entre el Estado y la sociedad, se explica por la confluencia de diferentes factores, entre los que se encuentran:

- El proceso de descentralización, que en el contexto de la reforma del Estado, llevó a la creación de nuevas estructuras político-administrativas en el ámbito subnacional con lo que se buscaba generar unas estructuras menos burocráticas.

- El acercamiento a la gestión de los programas al usuario de los servicios, introduciendo mecanismos de participación que posibilitaron la interacción entre los gobiernos locales y la población (aspectos estos que se han venido concretando en las distintas entidades federales con mayor o menor intensidad).

- El surgimiento de actores emergentes que han dado lugar a que se generen nuevas formas de organización desde la sociedad y que interaccionen con el Estado así como los gobiernos regionales buscando definir acciones en los distintos ámbitos. - El establecimiento de nuevo balance de poder que permite el desarrollo de una estructura organizacional subnacional, a partir de la cual los gobiernos regionales adelantan las políticas en este ámbito.

Formando parte de la reforma del Estado, fue adquiriendo fuerza preponderante la idea que éste no puede encargarse de todas las demandas provenientes de la multiplicidad de sectores sociales, por cuanto rebasa sus capacidades. En esta dirección se prevé que pueda apoyarse en las organizaciones de la sociedad para hacerle frente a las demandas 
globales y diversas, por medio de la participación de ellas en la ejecución de algunos programas sociales de carácter público.

Igualmente, organismos internacionales como el Banco Interamericano de Desarrollo (BID) y el Fondo Monetario Internacional (FMI), entre otros, frente al visible protagonismo de las organizaciones de la sociedad civil, buscan influir en los Estados Latinoamericanos para que recurran a dichas organizaciones para la gestión de lo social como instancias garantes de que los recursos puedan ser administrados con mayor transparencia. Es así como por esta doble condición, la interna y la externa, el Estado se ve en la necesidad de adoptar mecanismos de enlace con las denominadas organizaciones del tercer sector.

Razonamientos similares con bastante anterioridad fueron esgrimidos por intelectuales y políticos pertenecientes a organizaciones como la Comisión Presidencial para la Reforma del Estado (COPRE, 1989:5)르, cuando se adelantó el proceso de descentralización, cuyo énfasis principal se centró en la necesidad de disminuir la carga burocrática del aparato estatal, transfiriendo algunas funciones a organizaciones de la sociedad civil, promoviendo su participación en la consecución de su propio bienestar.

Cuando el Estado recurre a estas organizaciones, conduce a abrir nuevos espacios de acción a la sociedad civil, esto ha traído como consecuencia el desarrollo de la tendencia privatizadora de los servicios públicos, algunos como ocurre en salud. Independientemente de que se asuma una postura sobre la descentralización favorable a ella o no, los gobiernos regionales han ido creando sus espacios de acción producto de las circunstancias económicas y políticas a partir del momento en el cual ellos adquieren autonomía a partir de la puesta en práctica de la Ley Orgánica de Descentralización y Transferencia de Competencias del Poder Público (28/12/89). Dio origen a que respondan por acciones que antes eran competencia del gobierno nacional y que ahora, con el avance del proceso de descentralización, los gobiernos subnacionales han tenido que hacerle frente a los déficits sociales y para atender problemas que se consideran prioritarios, de allí que, estas organizaciones de la sociedad civil asuman un rol más activo a partir de dicho proceso así como en el contexto de la situación de crisis económica y social, generándose un incremento de su legitimidad en diversos espectros de la sociedad venezolana.

Es así, como a finales de la década de los ochenta, en el marco de las políticas de ajuste, se propone una política social que busca la eficiencia por medio de la complementariedad de acciones e incorporación de nuevos actores en fases fundamentales de la ejecución de las políticas sociales, como es el caso de la utilización de mecanismos que permitan el 
desarrollo de la acción focalizadora para identificar a los grupos que se hayan en condiciones de extrema pobreza. En este sentido, las políticas sociales son reorientadas hacia los grupos más vulnerables, el apoyo a los grupos de autoayuda, la promoción de la participación de la comunidad organizada en la gestión de los programas sociales y la incorporación de organizaciones de la sociedad civil en la provisión de los servicios sociales en cogestión con el Estado.

Las organizaciones civiles comienzan a ser concebidas como "socias" en la ejecución de programas sociales en cooperación con el Estado. Se intenta crear nuevas redes de atención a través de las organizaciones del Tercer Sector; estas entran en escena como proveedoras de servicios en cooperación con el Estado por el aumento considerable de la pobreza, pretendiendo mejorar las condiciones materiales de vida de los grupos más necesitados. Varios programas públicos comienzan a ser ejecutados mediante convenios con asociaciones civiles; surgen programas sociales, que si bien tienen un carácter compensatorio, se plantea concederle un rol protagónico a la sociedad civil por medio de organizaciones sociales, en la definición de prioridades y de soluciones a optar para proveer bienes y servicios.

La participación de las organizaciones del tercer sector en la ejecución de los programas, permite reducir los costos de ejecución, lograr una administración más autónoma y estimular, en las comunidades, la posibilidad de generar un sentido de pertenencia clave para el éxito y sustentabilidad de los programas (Machado, 1998:209). Esta participación de las organizaciones como ejecutoras de programas sociales tiende a que sea más eficiente y accesible la oferta de servicios a los grupos que tradicionalmente estaban excluidos. Así mismo, la relación que las mismas establecen con los beneficiarios de los programas, intenta romper con el carácter asistencialista atribuido a la política social, al visualizarlos como sujetos de su propio desarrollo.

A pesar de lo antes mencionado, muchos proyectos fracasan por la persistencia, tanto en el Estado como en los agentes públicos regionales, de la idea que era el gobierno quien debía resolver los problemas sin contar con el involucramiento de los sujetos así como por el sentido paternalista que animó la actuación del Estado; esto aunado a la ausencia de estudios que revelaran cuál era la representación social que los destinatarios de los programas tenían de dichos problemas y su manera de enfrentarlos.

Al iniciarse la incorporación de estas organizaciones en la gestión de los programas sociales, el Estado comienza a delegar funciones de rectoría y ejecución, específicamente en programas como el de Microempresa, Multihogares de Cuidado diario y el de Comedores Populares $\underline{3}$ sin preocuparse por mantener idóneos mecanismos de control, 
monitoreo y evaluación de impacto.

En los noventa4, se produce un aumento significativo de las organizaciones de la sociedad civil para enfrentar el problema de la pobreza, se comienzan a desarrollar estrategias para la medición y enfrentamiento de la misma. Muchas de esas organizaciones, se consideran a sí mismas como respuestas autónomas y espontáneas de las comunidades a la situación de pobreza, de allí que proponen alternativas para que la población solucione sus más urgentes problemas de sobrevivencia (Navarro, 1994: 91).

El problema de la pobreza comienza a ser considerado como cuestión relevante en América Latina, convirtiéndose en un tema prioritario dentro de las agendas de los gobiernos y ejes de las políticas que éstos dirigen. La revalorización de dicha temática tiene sus antecedente en los planteamientos realizados por la Comisión Económica para América Latina (CEPAL) sobre la llamada década perdida, la cual comprende esos diez años durante los cuales la región se encontraba sumergida en una profunda crisis relacionada con el agotamiento del Modelo de Industrialización Sustitutiva de Importaciones (Silva, 1999:91).

Las mediciones que se han realizado sobre la pobreza en Venezuela entre los organismos público y privados, indican que para 1993 el porcentaje de hogares en situación de pobreza era del 41\%, incrementándose significativamente para 1997 a 65\%; igualmente es significativo indicar el ingreso real de los hogares para ese mismo período, se redujo en un 70,9\% (Schiesser, citado por Silva, 1999:95). Estos datos evidencian la significación que ha adquirido el tema de la pobreza, por lo que la orientación de las políticas públicas es dar respuesta a este problema por medio de programas que contribuyan a su mitigación. De igual manera, las organizaciones del tercer sector promueven iniciativas que apuntan hacia esta dirección, como son algunas experiencias desarrolladas en la región zuliana como: el "Programa Socio Económico Sierra de Perijá", el Programa "Cambia un Rancho por una Vivienda Digna", y el "Centro Infantil Laguna de Sinamaica" (Comedores Populares) en el municipio Páez. En dichas iniciativas intervienen como socios el gobierno regional, iglesia, universidad, grupos comunitarios de base e instituciones internacionales aportando financiamiento, recursos humanos, logística, entre otros. Lo descrito confirma que la acción de las organizaciones civiles efectivamente alivian la carga financiera del gobierno central; aunado a esto recibe la relativa ayuda de las acciones por parte de las microempresas que, indirectamente, atacan el problema de la pobreza por cuanto procuran la capacitación, la generación de empleo y la diversificación de oportunidades productivas. 


\section{Tercer Sector, algunas precisiones teóricas}

Conceptualmente el término Tercer Sector es ambiguo, ya que mantiene algunos núcleos de imprecisión que bloquean la posibilidad de un uso más universal, pues al tratar de encontrar en la realidad los referentes concretos de actuación, de un gran número de organizaciones que buscan atender diversas necesidades que el Estado ya no puede satisfacer, o que surgen de la propia dinámica de acción societal, nos encontramos con un universo tan diverso aún en aquellos casos de incipiente presencia de estas organizaciones como es el caso venezolano.

Para algunos autores, el intento de puntualizar en qué consiste el tercer sector, ha generado controversia y la posibilidad de precisar sus características, en términos de definir los componentes que lo constituyen. Así tenemos que para Villasante (1997), el tercer sector, no va más allá de constituir un elemento "comparsa" al lado del Estado y el capital, no solo por lo inadecuado de su definición, sino también porque no contiene principios diferenciadores, ni muestra de identidad que den cuenta con propiedad, de su especificidad, y que trascienda el hecho de ser un elemento complementario del mercado o del Estado.

Para otros autores no reviste mayores problemas, tanto su conceptualización como la identificación de su rol en el conjunto de lo que denominamos sociedad civil; empero, se reconoce que tiene un carácter residual que le da cierta imprecisión. En algunos países de tradición anglosajona, el tercer sector se define como asociaciones sin fines de lucro, llamadas las "non profit organizations", en los Estados Unidos como "voluntary organizations" o “ sector voluntario" (Richer, 1997). Está relativamente claro, que el tercer sector se encuentra conformado por todas aquellas organizaciones que tienen como propósito desarrollar estrategias alternativas solidarias para la producción y provisión de servicios que tienden al desarrollo social, la promoción de la participación comunitaria, la lucha por la defensa de los derechos sociales en general y el sostenimiento del medio ambiente en las diversas áreas de desenvolvimiento de la vida del ser humano.

Independientemente de las disputas de académicos e intelectuales, las organizaciones del tercer sector han cobrado notoriedad y visibilidad, como una dimensión importante dentro de la sociedad civil. En el caso de Venezuela a finales de la década de los ochenta, con la conformación del orden democrático, la sociedad civil tuvo un papel poco preponderante en la estructuración del sistema político, explicado por el gran peso que los partidos políticos $\underline{5}$ y otras organizaciones de representación política tuvieron mayoritariamente en la década de los sesenta y setenta (Arenas, 2001:195), por lo que 
hay que esperar hasta la década de los ochenta cuando comienzan a surgir organizaciones de la sociedad civil, como opciones estratégicas importantes que se mueven en el escenario político, tales como: "Queremos Elegir" y COFAVIC (las cuales en los actuales momentos han desvirtuado la finalidad con la que originalmente fueron creadas, convirtiéndose finalmente en partidos políticos), y "Sinergia" que tiende a responder a enfoques democratizadores y participativos de la misma (Arenas, 2001: 197).

La visibilidad de las organizaciones del tercer sector en el campo social está relacionada con varios aspectos, entre los que se destacan los siguientes:

a) La fuerte presencia que estas organizaciones han logrado tener en Europa, Estados Unidos y en algunos países de América Latina, donde se estima que existen aproximadamente entre treinta y cinco mil y cincuenta mil organizaciones no gubernamentales dedicadas al trabajo con mujeres en muchos barrios marginales; por su capacidad de convocatoria, movilización, sentido de pertenencia y disciplina de trabajo.

b) Son organizaciones de lucha y defensa de los derechos e intereses de trascendencia universal.

c) Manejo adecuado de los recursos y capacidad para conseguirlos sistemáticamente.

d) Su capacidad para la replicabilidad y el éxito en los proyectos que emprenden, entre otros factores.

Sin embargo, existen algunos autores que no comparten esta versión tan maravillosa y romántica de las actividades de las ONG's; en particular Petras (2001) sostiene que tanto los regímenes Neoliberales como el Banco Mundial y otras instituciones del capital financiero interesadas en aupar el mercado, cooptarán y alentarán a las ONG's para socavar al Estado de Bienestar al suministrar servicios sociales para compensar los efectos de las políticas de ajuste promovidos por esas organizaciones de cooperación multilaterales, por lo que el término no gubernamental, lo que implica es desarrollar actividades que disminuyan el gasto público, liberando así un importante porcentaje de los fondos públicos para que estos puedan ser usados en beneficio de los capitalistas exportadores.

En el caso venezolano podemos acotar que esa imagen generalizada de actuación de las organizaciones del tercer sector, ha servido para que se recurra a ellas en el contexto de la crisis, siendo promovida esta iniciativa por las organizaciones financieras internacionales; en tal sentido cabe puntualizar que algunos autores señalan (Navarro, 1994; Machado, 1998), que:

a) En el contexto de la crisis el gobierno busca apoyarse en las ONG's y demás 
organizaciones que contribuyen a soportar los costos de proveer los servicios sin las limitaciones que impone una carga burocrática creciente.

b) El impulso dado por los organismos internacionales, los cuales plantearon que los recursos podían ser manejados con mayor transparencia y flexibilidad, debido a las ventajas que sus miembros tienen por la relación que ellos mantienen con las comunidades en donde actúan.

c) Por las necesidades de construcción de espacios nuevos de actuación, producto de la complejización, que ha adquirido la dinámica social en los últimos años, generando no sólo la presencia de nuevos problemas sino también por las necesidades que se han ido dibujando en el conglomerado social local.

Cabe destacar, sin embargo, que en Venezuela se ha registrado la constitución de organizaciones que fueron conformadas antes de la crisis de los ochenta, y que han desempeñado un rol muy destacado, este es el caso de una organización a nivel nacional, el Centro al Servicio de la Acción Popular (CESAP), que fue fundada hace más de 25 años, su nombre está articulado a la idea de lo popular, como una expresión de lo que significaron los movimientos sociales de la década de los 60, que guardaban correspondencia con un eje político-ideológico de izquierda, asociado a la teología de la liberación y a los movimientos eclesiales de base, que fueron beligerantes y posteriormente han ido decantando hacia acciones menos politizadas y orientadas hacia aspectos relacionados con problemas más puntuales vinculados con las necesidades insatisfechas y la pobreza.

En este sentido, es importante señalar, que en estas organizaciones ha existido una tendencia democratizadora, al promover la participación por medio de la conformación de estructuras más horizontales y aplanadas, que han iniciado diversas acciones encaminadas hacia el logro del desarrollo social. Así, encontramos ciertas agrupaciones fuertes y consolidadas, como CESAP, antes mencionada, que además de tener cierta incidencia en los programas sociales, también responde a un esquema de participación interna de sus miembros en el proceso de toma de decisiones tal como reconocen Salamanca (1993) y Chacín (2001).

A pesar de la ambigüedad del uso del término tercer sector, lo consideramos apropiado para captar una realidad que está surgiendo con fuerza en el acontecer socio histórico venezolano y ha ido adquiriendo significación política y social tanto, desde el punto de vista del análisis político como del sociológico. Así mismo, debe prestársele mayor atención al surgimiento, presencia y decantación de estas organizaciones, para poder diseñar una batería conceptual, que permita analizar más detalladamente el rol del 
Estado, su relación con estas organizaciones, y así poder definir cual es el Estado que necesitamos y el tamaño que éste debe tener.

Es imprescindible despejar ciertas dudas acerca de las fronteras relacionales entre Estado/ sociedad, Estado/ mercado, y sociedad/ mercado, así como también los aspectos relacionados con la descentralización, democratización, la flexibilización y responsabilidad social que conlleva los ímites del papel de éstas organizaciones en la realidad social venezolana.

En Venezuela, a diferencia de otros países, no ha existido una fuerte tradición no gubernamental, hecho que en principio puede atribuirse a la convergencia de dos factores vinculados con el comportamiento del Estado. El primero de ellos relacionado con el uso dado a la renta petrolera, que hizo que el Estado se convirtiera en el gran redistribuidor del ingreso generado $\underline{6}$, y el otro, vinculado con el fortalecimiento adquirido por el Estado en el proceso de consolidación democrática que ha perfilado el marcado intervencionismo estatal en el proceso de organización de la sociedad, teniendo también presente la excesiva injerencia de los partidos políticos en la conducción de las organizaciones que fueron emergiendo de la sociedad civil.

Aún teniendo en cuenta estas características, podemos indicar que el surgimiento de estas organizaciones es variado y que empezó a tener lugar concomitante al establecimiento del proceso democrático. Sin embargo, su fuerte incremento ha estado vinculado a la crisis que se vive en el país desde los inicios de la década del ochenta e impulsado más recientemente por las políticas de los gobiernos regionales en el contexto de los procesos de reforma del Estado y la descentralización.

Compartimos adicionalmente, la tesis de Navarro (1994:165) cuando indica que el nacimiento y multiplicación de las ONG's es reciente, gradual y se trata de un proceso inconcluso (Navarro, 1994:165) porque se encuentra en un abierto proceso de expansión, que viene teniendo lugar desde la conformación de los grupos de vecinos en los inicios del período democrático, para luego surgir los grupos de acción popular en la euforia de los años sesenta donde el movimiento social alcanzó bastante resonancia, movilizando a la gente a participar no sólo en acciones políticas sino culturales de confluencia de las iniciativas de protesta social y las acciones políticas que fueron muy fértiles en esos años, por lo que había despertado la revolución cubana.

En estos años se conocen iniciativas también asociadas con la liberación femenina, que procura fortalecer las organizaciones de tipo feminista; las ecológicas que se 
fundamentaban en los movimientos ambientalistas y las religiosas cristianas, que consolidan los grupos eclesiales de base, estimuladas por la influencia que ejerció la Teología de la Liberaci ón en el ámbito latinoamericano.

\section{Las Organizaciones del Tercer Sector estudiadas}

Para analizar la gerencia en las organizaciones del tercer sector en el municipio San Francisco del Estado Zulia, se seleccionaron, en función de su trayectoria histórica y la diversidad de sus programas y/o actividades, a tres organizaciones; el Equipo de Formación, Información y Publicaciones (EFIP), la Asociación Civil de Damas Salesianas y la Asociación Civil Nuevo Amanecer.

EI Equipo de Formación, I nformación y Publicaciones (EFIP), fue fundado en la ciudad de Valencia en 1972, registrado legalmente en 1976, por militantes de la juventud Obrera Católica, como un instrumento de apoyo educativo e informativo para el trabajo sindical y cultural con los jóvenes. Su objetivo está dirigido a diseñar programas de formación para jóvenes no escolarizados, en edades comprendidas entre 16 y 24 años, con poco o ninguna formación, ni experiencia laboral, que quieran ingresar al mercado de trabajo, formando microempresas o empleándose en oficios diversos (EFIP, 2002).

Para ello, el EFIP, diseña programas de capacitación y recurre a las microempresas para lograr la inserción de los jóvenes. Es importante mencionar, que a éstos jóvenes de escasos de recursos, se les asigna una beca que les permitía sufragar los gastos del transporte para asistir a los cursos de formación. El EFIP, inserta a los jóvenes para que realicen sus pasantías en microempresas de la comunidad, realizando para ello, una campaña de sensibilización a los microempresarios para el proceso de inserción laboral e igualmente, el EFIP, posee otros programas, como el de atención al microempresariado, información juvenil, apoyo, creación y fortalecimiento de iniciativas emprendedoras, apoyo a la microempresa juvenil, atención a las organizaciones de la comunidad.

En los últimos diez años, esta organización a reportado una importante labor, al contribuir a formar a más de veinte mil personas en todo el país en la formación para el trabajo. En cuanto a las fuentes de financiamiento, recibe apoyo de entes internacionales, tales como; Inter-American Foundation, Ayuntamiento del Logroño y la Comunidad Autónoma de La Rioja (España), y de empresas nacionales e internacionales, como Brot (Fui die Welt).

Por otra parte, se cuenta con la Asociación Civil Nuevo Amanecer, fundada en 1985 
y registrada legalmente en 1992, se encuentra asociada al grupo social CESAP (Centro al Servicio de la Educación Popular). Su objetivo es contribuir al mejoramiento de la calidad de vida de las comunidades de la región zuliana con el apoyo de organizaciones gubernamentales y no gubernamentales. La asociación desarrolla una variedad de proyectos en cinco áreas, como son: apoyo a la gestión económica; Justicia de paz; desarrollo humano; desarrollo y calidad de vida y gerencia social, donde se tienen proyectos como asesoría para el desarrollo organizacional, liderazgo en la gerencia comunitaria y capacitación a instituciones También desarrolla actividades como; talleres, cursos y charlas informativas dirigidos a la comunidad en general (Nuevo Amanecer, 2002).

En lo referente a las fuentes de financiamiento Nuevo Amanecer ha recibido el financiamiento para la ejecución de su proyecto de parte de instituciones públicas o privadas, como Petróleos de Venezuela y sus Empresas Filiales (PDVSA) Alcaldías, el Consejo nacional de Cultura (CONAC), la Fundación FORD y Carbones de Guasares.

Así mismo, existe la Asociación de Damas Salesianas, conformada por mujeres Católicas laicas, fundada en 1968 y de irradiación nacional, con sede en el Municipio San Francisco. Desarrollan programas sociales en las áreas de salud, educación para el trabajo y promoción humana integral, dirigidos hacia los sectores más necesitados (Damas Salesianas, 2002).

En el Estado Zulia, esta organización, administra algunos ambulatorios cuya infraestructura pertenece al Sistema Regional de Salud, como es el caso del ambulatorio urbano nivel I, en San Jacinto, tienen la responsabilidad de administrar el Ambulatorio Don Bosco, el cual presta nueve servicios: medicina general; cirugía general; oftalmología; traumatología; odontología; nutrición y dietética; psicología, ginecología, y servicios especiales. En el área de educación cuenta con una unidad educativa, que atiende a niños en edades preescolar y primaria, dicta cursos de capacitación para jóvenes y adultos, entre 16 y 20 años. Tiene un centro de capacitación, denominado La Chinita, por medio de una alianza estratégica con el Instituto Nacional de Capacitación Educativa (INCE). En el área de formación integral del individuo, realizan un conjunto de actividades destinadas a la formación cristiana, acompañadas de actividades deportivas y actividades de habilidades de desarrollo personal y deportivas, con el apoyo de grupos de béisbol profesional.

Con respecto a las fuentes de financiamiento tenemos, que reciben el apoyo de diversas organizaciones públicas y privadas entre las que se encuentra: Gobernación del estado 
Zulia y Alcaldías de Maracaibo y San Francisco, Hotel del Lago, Fundasalud, Fundación Polar, Fundación Eugenio Mendoza y la Fundación del Niño; Empresas privadas como; Casa París y Chevron; y los diarios Panorama, Regional y el Heraldo. Esta organización, tiene presencia organizacional en veintiséis naciones, entre la que destaca: Estado Unidos, México, Colombia, Ecuador, Perú, Argentina, España y Japón, y cuenta con más de 150 centros.

En la dinámica de actuación de estas tres organizaciones del tercer sector, se expresa una diversidad de formas de gestión de proyectos y programas, que pueden ser diseñados o elaborados por ellas o también pueda presentarse la cogestión de proyectos y programas de instituciones del sector público o privado; en este aspecto el EFIP representa una organización que formula y desarrolla programas de capacitación e inserción laboral en forma independiente propiciando luego el apoyo del sector privado básicamente representado por las microempresas. En el mismo orden de ideas, las Damas Salesianas se perfilan como el tipo de organización que prefiere actuar en forma mucho más independiente y diferenciada del Estado, por cuanto consideran que este ente debe ocuparse de las funciones que son de su exclusiva responsabilidad, por ello manifiestan el resguardo de sus niveles de autonomía y por ende lo limitado de su interacción con este ente. En Nuevo Amanecer se configura una posición mixta, dado que llevan a cabo algunos proyectos y programas ejecutados en forma autónoma en catorce Municipios del Estado Zulia, pero también algunos otros ejecutados en forma compartida con diversas instituciones como, la Alcaldía del Municipio Maracaibo, La Universidad del Zulia (LUZ), el Programa de Vivienda de Interés Social (PROVIS) entre otras, con el apoyo también de proyectos y programas con empresas como PDVSA y Carbones del Guasare. Nuevo Amanecer, entre el 01 de Enero y el 20 de Septiembre del 2001, gestionó el otorgamiento de 850 micro créditos, por medio del programa de apoyo a la economía popular.

\section{La Gerencia en las organizaciones del Tercer Sector}

Para analizar la gerencia de las organizaciones objeto de estudio, es necesario describir la estructura administrativa, los procesos administrativos, comunicacionales y de toma de decisiones, lo cual permitirá conocer el funcionamiento de la gerencia y los posibles problemas que les afectan.

\subsection{Estructura Organizativa}

La estructura organizativa es la forma como esta organizada una empresa o institución, es decir, la división de funciones o actividades y la interrelación de las partes que la 
conforman, indica, también, la jerarquía, autoridad, así como las relaciones de subordinación (Hall, 1989:52).

Para algunos autores, la estructura administrativa, esta conformada por una esfera política y una esfera tecno-administrativa; la primera denominada, también, decisional, es el espacio donde los altos ejecutivos y administradores toman las decisiones fundamentales de la organización. La esfera tecno-funcional, es el espacio donde se concentran los planes y actividades operativos de la organización (Briceño, Machado y Rodríguez, 1997). Es importante destacar, que entre estas dos esferas existe una instancia bisagra denominada gerencia, que es la responsable del cumplimiento de los objetivos planificados en la esfera política.

En cada una de las esferas se desarrollan diferentes procesos administrativos, a la esfera política le corresponde definir las orientaciones estratégicas de la organización y lo relativo a la planificación, dirección y control, a la instancia gerencial, le compete el cumplimiento de los objetivos, la operacionalización de los planes, programas y actividades, de acuerdo a las directrices fijadas en la instancia política y gerencial (Hall, 1989:53).

En este sentido, encontramos en las organizaciones estudiadas, una variada gama de formas organizativas que responden a esquemas que van desde formas más complejas y diferenciadas a otras que se caracterizan por contar con una estructura más sencilla y poco diferenciada. En el caso del Equipo de Formación Información y Publicaciones (EFIP), cuenta con un grupo coordinador integrado por cuatro miembros y una asamblea que funciona a nivel nacional, integrada por veinte miembros, con una estructura, compuesta por un presidente nacional, un secretario y un tesorero, que constituye la directiva legal y quienes definen las orientaciones estratégicas de la organización a nivel del estado Zulia, poseen una estructura organizacional, conformada por una dirección ejecutiva y una dirección coordinadora, esta instancia tiene una relativa autonomía. La esfera política, es la denominada dirección ejecutiva, es la instancia donde se toman las decisiones fundamentales del Equipo de Formación y Publicación, integrada por cinco miembros, quienes dirigen y controlan las acciones estratégicas de dicha organización. Así mismo, se plantean estrategias para establecer alianzas con otras organizaciones gubernamentales y no gubernamentales, nacionales e internacionales, así como, la empresa privada, para fortalecer los programas de capacitación. La dirección coordinadora, como instancia gerencial, planifica, coordina y controla lo referentes a los talleres de capacitación en las áreas de mecánica automotriz, dirigidos a los jóvenes de la comunidad. Establece relaciones de cooperación con empresas y microempresa de la localidad de San Francisco para que los jóvenes realicen sus pasantías de estudios. 
La Asociación Civil Nuevo Amanecer, posee una estructura integrada por 23 miembros en los grupos que funcionan en el Municipio San Francisco y La Costa Oriental del Lago. La instancia decisional de la Asociación es el Comité Directivo conformado por siete miembros, encargados de fijar los lineamientos de acción teniendo como base las orientaciones estratégicas que rigen toda la organización, establecer alianzas con otras organizaciones públicas y privadas y generar alternativas de financiamiento. La gerencia de la Asociación Nuevo Amanecer, es la Coordinación Ejecutiva, encargada de planificar los programas y proyectos en las diferentes áreas, mientras que la instancia tecnofuncional, esta conformada por trece unidades operativas encargadas de ejecutar los programas de acuerdo a las diferentes áreas.

La Asociación Civil Damas Salesianas, tienen una estructura organizativa centralı̄ inferimos que más compleja que las dos anteriores, dado que esta asociación en el área de salud, administra ambulatorios tipo II del Sistema Regional de Salud en el estado Zulia, dichos ambulatorios poseen una estructura administrativa propia. En el caso del ambulatorio Don Bosco la esfera de las decisiones es manejada por la Asociación de Damas Salesianas, y la instancia gerencial es la denominada dirección asistencial del ambulatorio, con once unidades operativas que ejecutan los programas de salud en diversas áreas.

El crecimiento y complejización de la estructura organizativa, no parece estar relacionada con el tiempo que éstas organizaciones llevan funcionando, esto se pone de manifiesto, cuando se observa por ejemplo el caso del EFIP; en el caso de Nuevo Amanecer, la complejización de la estructura organizacional, están asociados con la ampliación de compromisos que se van a adquirir con los proyectos y programas que van surgiendo, la ampliación del número de miembros, a la expansión del radio de acción y al fortalecimiento de la organización por la vía del crecimiento de sus fronteras de actuación.

\subsection{Procesos administrativos}

Los miembros que forman parte de una determinada esfera de la organización asumen funciones o responsabilidades que varían de acuerdo a la esfera en que se ubique, estas funcionen son los denominados procesos administrativos entre los que encontramos: la planificación, dirección y control, los cuales se generan simultáneamente con los procesos comunicacionales y de toma de decisiones, sin embargo, acá no se pretende evaluar el funcionamiento de estas organizaciones, sino presentar los casos. 
La planificación: es un proceso que se lleva acabo en la esfera política o decisional de una organización, y se refiere a la forma como los gerentes de una organización fijan las metas y estrategias adecuadas para el logro de dichas metas (Stoner, 1989:169).

En los casos de las organizaciones estudiadas, tenemos que han fundamentado sus acciones en función del cumplimiento de objetivos y metas, enfocados en diversas áreas, cabe destacar que esa planificación es flexible, adaptada a la dinámica de la realidad, tomando en cuenta el surgimiento de nuevas demandas en las comunidades y nuevos financiamientos para atacar problemáticas específicas. La Asociación Civil Nuevo Amanecer, le da más formalidad a sus acciones, planificadas en función de procedimientos y agenda de actividades; mientras que el EFIP y Damas Salesianas, manejan un nivel de formalidad menor, donde la consulta a la comunidad, en algunos casos, es tomada en cuenta en el momento de planificar. Las tres organizaciones desarrollan sus actividades en función de estrategias y tomando en consideración los actores aliados y oponentes de las acciones a emprender.

Siguiendo las pautas modernas de análisis organizacional, se encuentra un dato de referencia importante, las organizaciones en estudio han aplicado el enfoque de Planificación Estratégica, que plantea la formalización de la misión, visión, objetivo y rol de la organización, donde es básico destacar el carácter de servicio y contribución al logro de objetivos de desarrollo que están llamadas a cumplir, expresado, tanto en la misión como en la visión; esto es significativo por el hecho de contribuir a fijar una directriz de orientación de la organización, un signo de identidad colectiva y una referencia de integración de sus miembros.

El Control: es un importante proceso que se ejecuta a nivel de la esfera política, según Stoner (1989:170), intenta medir el avance en el cumplimiento de metas fijadas, al mismo tiempo detectar las desviaciones que se presentan en la ejecución de los planes y aplicar los correctivos necesarios. De allí, que se encuentra en estrecha relación con la planificación y la comunicación, ya que en la medida que haya retroalimentación entre los miembros de la organización, en esa medida se puede saber como está funcionando la organización.

En las organizaciones en estudio se realiza la evaluación y el control de los programas y actividades de manera permanente, por medio de la comunicación que se establece entre los miembros. El EFIP y Damas Salesianas, llevan un registro sistematizado de las actividades realizadas, los resultados esperados y alcanzados, y la comunicación entre las unidades operativas y la gerencia sobre el desarrollo de los programas y/o actividades 
permite que se realice los correctivos que sean necesarios. La consulta a los beneficiarios de los programas de capacitación de EFIP y de salud de Damas Salesianas es vital para realizar los ajustes y correctivos para lograr una mejor eficacia y eficiencia de los mismos. La Asociación Civil Nuevo Amanecer, realiza evaluación de sus actividades en función de los resultados fijados y obtenidos, tomando en consideración la opinión de los beneficiarios de los servicios. Esta Asociación realiza un seguimiento permanente al manejo de los recursos de los programas, ya que rinden cuentas a las instituciones que financian algunos programas y proyectos, como el Ministerio de la Familia, presentando un informe trimestral de rendición de cuentas.

Toma de Decisiones: es entendido como la forma en que los miembros directivos de la organización planifican las acciones a seguir y las estrategias a implementar para ello; este aspecto resulta significativo en el análisis de la gerencia, convirtiéndose en un eje central del estudio. En el tipo de organizaciones donde se supone impera un espíritu democrático, el proceso de toma de decisiones debe corresponder a un estilo flexible, abierto y participativo, según Gordon (1997), debe contribuir al fortalecimiento de los valores de tolerancia, pluralidad, respeto y solidaridad.

En los casos seleccionados, se registra que la instancia de toma de decisiones es la Asamblea - la cual está constituida por un número significativo de sus miembros- utiliza los mecanismos de consulta directa por votación (en el único caso en el que esto no pudo ser corroborado fue en el de las Damas Salesianas).

Hasta ahora los casos observados muestran una tendencia hacia la democratización del proceso de toma de decisiones, ya que se ha podido visualizar que en el mismo intervienen otros miembros de la organización quienes en conjunto conforman la asamblea, como un mecanismo de consulta directa, sin embargo, cabe acotar que estas decisiones siguen siendo, en la generalidad de los casos, un proceso interno de la organización, con muy escasa participación de los miembros de la comunidad a los cuales van dirigidos los programas; sólo en el caso de Nuevo Amanecer 8 son tomados en cuenta otros actores o instituciones por la característica de flexibilidad con que esta organización funciona.

Otro aspecto importante de señalar, se encuentra relacionado con el clima organizacional, que tiende a basarse en un espacio de comunicación y participación entre los miembros, hasta el punto que se consideran sus opiniones y sugerencias en la toma de decisiones, lo cual ha generado un compromiso, sentido de pertenencia e identificación con las organizaciones a las cuales pertenecen, lo que se convierte en una fortaleza, pues 
se sienten parte de ellas, garantizando su permanencia y propiciando de esta manera un espacio para la participación.

\subsection{Proceso Comunicacional}

La comunicación como un aspecto inherente a los procesos gerenciales es por definición, según Hall (1989), el proceso de intercambio de información y transmisión de significados entre las partes que conforman una organización.

En este sentido, las formas de comunicación en las organizaciones estudiadas son tanto orales como escritas. En EFIP, Nuevo Amanecer y Damas Salesianas, se realizan reuniones periódicas y asambleas para someter a discusión tópicos de interés, realizar evaluaciones de los programas y establecer nuevas estrategias de financiamiento, lo cual facilita el proceso de retroalimentación entre los diversos niveles jerárquicos. Los responsables de los programas y actividades, mantienen una comunicación directa con los coordinadores de la instancia decisional, que tiende hacia la horizontalidad y permiten potenciar el proceso de control de las decisiones sobre los programas, proyectos que desarrollan las organizaciones.

Un último aspecto importante para la gerencia son las estrategias utilizadas para captar nuevos miembros, en las tres organizaciones, se evidencian mecanismos muy semejantes en relación al proceso de incorporación de los miembros aún y cuando existan algunas diferencias en términos de los esfuerzos realizados para su incorporación. El EFIP, mantiene una actitud pasiva, es decir, esperan que los sujetos interesados se acerquen a la organización y manifiesten su deseo de formar parte de este equipo; las Damas Salesianas se caracterizan por ser más activas, por cuanto realizan campañas permanentes para el reclutamiento de nuevos miembros (en este caso mujeres voluntarias), como también la elaboración de abundante promoción con la finalidad, por una parte, de mantener informados y motivados a sus miembros y por la otra, contribuir a mantener en el tiempo el trabajo voluntario de estas mujeres. Estas acciones son menos evidentes en los casos de Nuevo Amanecer y el EFIP, al realizarles las entrevistas, reconocen la inexistencia de campañas definidas para la captación de nuevos miembros.

\subsection{Modalidades de participación}

Las organizaciones en estudio presentan modalidades de participación muy homogénea. La Asociación Civil Nuevo Amanecer, el EFIP y Damas Salesianas desarrollan estrategias para promover la participación de sus miembros por medio de mecanismos como las 
asambleas y reuniones periódicas. Esta modalidad participativa es de tipo consultiva o asesora, tiene un carácter de influencia en la decisión, y se expresa como la opinión sobre las decisiones que se adoptaran. Así planteada este tipo de participación se encuentra presente en estas organizaciones, dado que las personas que dirigen las organizaciones tienden a incorporar con frecuencia la opinión del resto de los miembros en los asuntos sometidos a decisión.

Este tipo de participación ha desarrollado a lo interno un sentido de pertenencia e identificación de los miembros para con las organizaciones, por la forma como se establecen las relaciones entre los diversos niveles jerárquicos, orientada hacia la horizontalidad y evidenciada en el respeto, la comunicación y la confianza.

\section{Consideración final}

En las organizaciones objeto de estudio, EFIP, Damas Salesianas y Nuevo Amanecer, se encuentran una diversidad en los componentes de la estructura funcional y en los procesos gerenciales. Es pertinente señalar que en ellas, coexisten algunos rasgos comunes que tienden a ampliar el sentido de participación, al incorporar a otros miembros en las asambleas realizadas, delegar funciones, y al romper con la comunicación vertical, lo que permite aprovechar las potencialidades del voluntariado y estimular a los distintos miembros de dichas organizaciones.

En el proceso de toma de decisiones, se adoptan dos estilos, en primer lugar, participativo, cuando los coordinadores de estas organizaciones en las decisiones toman en cuenta las opiniones y/o sugerencias del resto de los miembros, en segundo lugar, no participativo, cuando los líderes de estas organizaciones, al tomar decisiones trascendentales o estratégicas no incorporan al resto de los miembros, por medio de mecanismos como la consulta. Con respecto al estilo participativo, que genera influencia en las decisiones, se realiza por medio de asambleas, reuniones periódicas de carácter informal entre sus miembros y en uno sólo de los tres casos, en algunas oportunidades se ha dado la incorporación de los miembros de la comunidad, al genera un contacto con los grupos organizados que sirven de intermediarios con las organización.

Con respecto al tipo de participación que se está dando en estas organizaciones, puede conjeturarse como beneficiosa para la organización, por cuanto fortalece el sentido de pertenencia e identificación de los miembros para con las organización, por la forma como se establecen las relaciones entre los mismos, orientadas hacia la horizontalidad, evidenciando un clima organizacional de respeto mutuo, confianza, apoyo y solidaridad, 
de allí que pueda decirse que en estas organizaciones se esta construyendo un sentido del "nosotros", los que algunos autores consideran un rasgo distintivo de capital social. Sin embargo, hasta donde se ha avanzado en la investigación con relacionado a la participación, se desprenden algunas interrogantes a ser corroboradas cuando se estudien los otros casos, que permitan profundizar el análisis; las inquietudes al respecto tienen que ver con: ¿Cuál es el estilo predominante de participación que se registra en estas organizaciones? ¿Cuáles son los mecanismos de participación más frecuentemente utilizados? ¿Cuál es el carácter que asume la participación de estas organizaciones en la provisión de servicios sociales en cooperación con el Estado? ¿Intervienen las organizaciones en el proceso de toma de decisiones de la ejecución de los programas sociales de carácter público?.

Por otro lado, se encontró que la comunicación entre los miembros de EFIP, Damas Salesianas y Nuevo Amanecer, tiende hacia un proceso de retroalimentación, expresado en los diálogos y conversaciones realizadas con frecuencia entre los diversos miembros, que se comienza a reconocer en atención a la necesidad de aglutinar esfuerzos sobretodo en materia de capacitación. En términos generales el proceso de comunicación en las organizaciones es bastante fluido y dinámico, se expande por las organizaciones, sin seguir reglas o patrones fijos, y aunque teóricamente existen niveles jerárquicos la comunicación tiende a estimular la horizontalidad.

Es importante mencionar que estas organizaciones poseen una diversidad en sus fuentes de financiamiento, al establecerse alianzas con organizaciones públicas y privadas, resaltándose las relaciones con organismos internacionales e instituciones del gobierno central, regional y local.

\section{Notas}

* Este artículo contiene los resultados preliminares del Proyecto de Investigación intitulado: “Organizaciones del Tercer Sector, Política Social y Gestión de Programas Sociales", adscrito al Centro de Estudios Sociológicos y Antropológicos (CESA) FACES-LUZ y contó con la colaboración de estudiantes de la Cátedra electiva “Organizaciones del Tercer Sector" dictada en la Escuela de Sociología FACES-LUZ. Dicho proyecto es financiado por el Consejo de Desarrollo Científico y Humanístico de LUZ (CONDES.

1. Ku Klux Klan, sociedad secreta que se originó en los Estados Unidos después de la guerra de secesión en 1867, con la finalidad de luchar en contra de la integración de los negros.

2. La COPRE fue creada en 1984 por el presidente Jaime Lusinchi y tuvo como sus encargos más importantes, 
elaborar un Proyecto de Reforma Integral del Estado, el cual tuvo una obvia y precisa dimensión política, cuyo sentido es perfeccionar los mecanismos de representación y de participación ciudadana, así como impulsar la democratización de los partidos políticos, contribuyendo a generar una institucionalización del Estado y un campo de acción genuino de la sociedad civil.

3. Para algunos autores la interacción entre el Estado y las organizaciones civiles en la provisión de servicios sociales, tiene como norte la privatización de las políticas sociales (Leal, 1996:83). Las ONG's asumen la responsabilidad de proveer servicios sociales y el Estado aunque continúa como el principal financiador de los programas sociales, pasa a un segundo plano como instancia central de la política social, aspecto este que será profundizado en posteriores análisis.

4. En el caso particular del estado Zulia, a partir de 1989 se produce un incremento significativo de las organizaciones del tercer sector vinculadas con el área de salud, deporte y cultura, muchas de las cuales fueron promovidas desde el gobierno central; en otros casos, la política del gobierno regional por medio de sus instancias locales han estimulado su surgimiento, como por ejemplo, el caso del Instituto Regional de Deporte del estado Zulia (IRDEZ), ha promovido la creación de más de veinte organizaciones en el municipio San Francisco y Maracaibo. Este Instituto desarrolla un programa denominado "Fortalecimiento de la Sociedad Civil", cuyo objetivo es orientar a la población en el proceso de conformación legal de las organizaciones sin ánimos de lucro en el área deportiva, como requisito exigido por esta institución regional, para brindar el financiamiento a este tipo de organizaciones por la vía de proyectos sociales. Por otro lado, encontramos organizaciones cuyo objetivo es combatir o aliviar la situación de pobreza existente en la región, tales como grupos femeninos, asociaciones de producción o de consumo, algunas de las cuales tienen un carácter benéfico o de filantropía social.

5. Al respecto ver el interesante trabajo de Coppedge (1993) “Participación y Reforma en una Perspectiva Comparada, en Venezuela: La Democracia bajo Presión. Instituto Venezolano de Estudios Sociales y Políticos y North Center de la Universidad de Miami.

6. Sobre la renta petrolera y su significado en el comportamiento del Estado, existen diferentes trabajos de Los cuales mencionaremos los de Bernardo Mommer (1997), “La Renta Petrolera, su Distribución en el Desarrollo del Capitalismo Rentístico Venezolano, ILDIS Caracas; Asdrúbal Baptista y Bernardo Mommer (1987), El Petróleo en el Pensamiento Económico de Venezuela. Un Ensayo. Ediciones IESA Caracas y Luis Pedro España (1987), Democracia y Renta Petrolera, Universidad Católica Andrés Bello, Caracas.

7. La información de la Estructura administrativa central de la Asociación de Damas Salesianas, no fue suministrada.

8. Flexible en el sentido de que en esta organización pueden formar parte de la directiva, miembros de otras organizaciones así como representantes de la sociedad civil, ellos indican no ser un ente cerrado sino que permiten la intervención de otros actores como La Universidad del Zulia, la Dirección de Desarrollo Social de la Gobernación del 
estado Zulia entre otros, con la finalidad de darle mayor dinamismo a su asociación, así como por la necesidad por una parte, de atacar o disminuir el punto de vista presidencialista o centralizado, y por otra, de buscar el consenso y la representación (Nuevo Amanecer, 2002).

\section{Bibliografía Citada}

1. Arenas, Ender (2001), “Política, Legitimidad y Democracia en una sociedad en Transición: Las Viejas y Nuevas Representaciones Sociales, sobre las nociones de Política, Partidos Políticos y la Sociedad Civil bajo Impacto de la Crisis Social Venezolana, Notas para su discusión". En Revista Espacio Abierto, Vol. 10 No. 2 /abril- junio, pp. 187-200.

2. Briceño, Machado y Rodríguez (1997), Modelos y Problemas Gerenciales de la Unidad de Desarrollo Local ( UNDEL). Mimeografiado. Maestría en Desarrollo Social. Cátedra Modelos gerenciales. División de Estudios para Graduados. FACES. Universidad del Zulia. Maracaibo.

3. Chacin, Ronald (2001), “Hacia una Evaluación del Pluralismo Democrático en Venezuela: Los Partidos Políticos y las Agrupaciones Sociales en el periodo 1989-1993". En Revista Espacio Abierto, Vol. 10 No. 2 /abril- junio, pp. 201 -227.

4. Congreso de la República de Venezuela. Ley Orgánica de Descentralización, Deslimitación y Transferencia de Competencias del Poder Público. Gaceta Oficial. No. 4153 de fecha 28 de Diciembre de 1989.

5. Coppedge, Michael (1993), "Participación y Reforma en una Perspectiva Comparada". En Venezuela: La Democracia bajo Presión. Instituto Venezolano de Estudios Sociales y Políticos, North-South Center de la Universidad de Miami. Serbin/Stambouli/McCoy/ Smith Editores.

6. Copre (1989), La Reforma en Síntesis. Documento de proyecto de reforma integral de Estado. Comisión Presidencial para la Reforma de Estado. pp. 71.

7. Damas Salesianas (2002), Entrevista efectuada a la Directiva. Febrero. San Francisco.

8. EFIP (2002), Entrevista efectuada al Coordinador del Equipo de Formación y Publicación. Marzo. San Francisco. 
9. García, J. y Sarcos, K. (2000), “Iglesia y Participación Comunitaria en Salud: el Caso de Municipio San Francisco del estado Zulia, Venezuela". En: Revista de Ciencias Sociales. Vol. 3 No. 1. Enero-Abril, pp. 75-94.

10. Gordon, Sara (1997), “La Cultura Política de las Organizaciones No Gubernamentales de México". En: Revista Mexicana de Sociología. Vol. 59 No. I enero-marzo 1997, pp. 53-67.

11. Hall, Richard (1989), Organizaciones, Estructuras y Procesos. Tercera Edición, México, pp. 353.

12. Leal, Nila (1996), “La Participación Comunal en Colombia y Venezuela" ¿Privatización o democracia Participativa? En Revista Venezolana de Gerencia, Vol.1, No.1.

13. Machado, Clemy (1998), “Los Gobiernos Locales: Participación Ciudadana y Democracia". En: Descentralización, Gobierno y Democracia Coordinador (Carlos Mascareño) CENDES, Caracas.

14. Navarro, Juan Carlos (1994), Las Organizaciones de Participación Comunitaria y la Prestación de los Servicios Sociales a los Pobres en América Latina. Ediciones IESA. Caracas, Venezuela, pp. 41-109.

15. Nuevo Amanecer (2002), Entrevista efectuada a la Directora Suplente de la Organización Civil Nuevo Amanecer. Enero. San Francisco.

16. Richer, Madeleine (1997), “Las Organizaciones del Tercer Sector, un nuevo Objeto para las Ciencias de la gestión". En: Revista Venezolana de Gerencia. Año 2, No. 3 pp. 77-91.

17. Rizzardini, Marco (1997), Tercer Sector, Voluntariado y ONG's. Una Reflexión crítica a partir de la experiencia de SODEPAZ. En: ¿Trabajo Voluntario o Participación? Elementos para una Sociología del Tercer Sector. Ariel Jerez (Coordinador). Editorial TECNOS, Madrid, pp. 256-257.

18. Rodríguez, I. y Pérez, J. (2000), “Descentralización, I glesia y Salud en la década de Ios 90: El Caso del estado Zulia, Venezuela". En: Revista Ciencias de Gobierno. Año 4 No. 7. Enero-Junio, pp. 41-62. 
19. Salamanca, Luis (1993), El Sistema Político Venezolano, una Lectura desde la Sociedad Civil. En: Venezuela: Democracia Bajo Presión. Instituto Venezolano de Estudios Sociales y Políticos, North-South Center de la Universidad de Miami. Serbin/Stambouli/McCoy/Smith Editores, pp 161-175.

20. Salinas R. Francisco (1997), El Estado de Bienestar y las Organizaciones Voluntarias. En: ¿Trabajo Voluntario o Participación? Elementos para una Sociología del Tercer Sector. Ariel Jerez (Coordinador). Editorial TECNOS, Madrid, pp. 109-134.

21. Silva M., Héctor (1999), La Política Social en Venezuela durante los años ochenta y noventa. Fondo de Inversión Social de Venezuela (FONVIS), Centro de Estudios del Desarrollo (CENDES). Editorial Nueva Sociedad, ILDIS pp. 86-114.

22. Stoner, James (1989), Administración, Editorial Hall hispanoamericana, tercera edición, México, pp. 773.

23. Villasante, Tomás R. (1997), Desde Las Redes Locales hacia un Tercer sistema Democrático. En: ¿Trabajo Voluntario o Participación? Elementos para una Sociología del Tercer Sector. Ariel Jerez (Coordinador). Editorial TECNOS, Madrid, pp.183-206. 